\title{
Nível de sombreamento, umidade do solo e morfologia do cafeeiro em sistemas agroflorestais
}

\author{
Renato Alves Coelho ${ }^{1}$, Sylvana Naomi Matsumoto², Carmem Lacerda Lemos ${ }^{3}$, Francisco Adriano de Souza ${ }^{4}$
}

\begin{abstract}
Com o objetivo de avaliar características morfológicas dos cafeeiros e a conservação de umidade do solo em diferentes sistemas agroflorestais, foi realizado este experimento. O ensaio foi conduzido no município de Barra do Choça-BA, em uma propriedade particular, sendo observadas três áreas de produção distintas: (1) cafeeiro cultivado a pleno sol (MONO), (2) cafeeiro conduzido em associação com o vinhático (SAF-CV) e (3) cafeeiro associado ao abacateiro e ingazeiro (SAF-CAI). Em março/2007, época úmida, e em agosto/2007, época com menor precipitação, foram realizadas avaliações relacionadas à incidência de luz (determinação do nível de sombreamento dos sistemas com base no percentual de radiação fotossintética incidente em relação à radiação fotossintética global), à umidade do solo (US) e à morfologia do cafeeiro (área foliar individual (AFI), altura e diâmetro do caule e da copa). Foi verificado intenso sombreamento para os SAFs (março, 93,3\% em SAF-CV e 82,3\% em SAF-CAI; e agosto, 37,5\% em SAF-CV e 89,5\% em SAF-CAI). Na época de menor precipitação, maior US foi observada para MONO (17,3\%), seguido por SAFCV (15,1\%) e SAF-CAI (9,5\%). Os sistemas de cultivo definiram os parâmetros morfológicos avaliadados do cafeeiro, sendo observada maior AFI para os SAFs nas duas avaliações realizadas.
\end{abstract}

Palavras-chave: Arborização, radiação fotossintética, Coffea arabica.

\section{RESUMO}

\section{Shading level, soil water content and coffee growth under agroforestry systems}

The objective of this study was to evaluate morphological characteristics of coffee plants and soil water conservation in different agroforestry systems (SAF). The experiment was carried out in Barra do Choça, Bahia, in a private farm. Three different coffee fields were evaluated: (1) in a full sun (FS); (2) intercropping with Planthaginea sp. (AFS-P) and (3) association with avocado and inga trees (SAF-AI). In March/2007, rainy season, and in August/2007, season with lower rainfall indexes, the following parameters were measured: (a) shading levels of the systems, considering the photosynthetic incident radiation in relation to the global photosynthetic radiation; (b) soil water content (SWC) in a depth of 0-20 cm; and (c) morphological characteristics of coffee plants (individual leaf area - ILA, height and diameter of stem and canopy). The agroforestry systems promoted a high shading index (93.3\% for AFS-P and 82.3\% for AFSAI, in March, and 37.5\% and 89.5\% for AFS-AI and AFS-P in August, respectively). In the dry season, a higher SWC was found for FS (17.3\%), followed by AFS-P (15.1\%) and AFS- AI (9.5\%). In all evaluations, ILA was higher in AFSs than in FS.

Key words: Shading, photosynthetic active radiation, Coffea arabica.

Recebido para publicação em novembro de 2008 e aprovado em outubro de 2009

${ }^{1}$ Licenciado em Ciências Agrícolas, Mestre. Universidade Estadual do Sudoeste da Bahia, UESB, Programa de Pós-graduação em Agronomia, Estrada do Bem Querer, km 04, Caixa Postal 95, 45000-000, Vitória da Conquista, Bahia, Brasil. renatoacoelho@yahoo.com.br.

${ }^{2}$ Engenheira-Agrônoma, Doutora. Universidade Estadual do Sudoeste da Bahia, UESB, Departamento de Fitotecnia e Zootecnia, Estrada do Bem Querer, Km 04, Caixa Postal 95, 45000-000, Vitória da Conquista, Bahia, Brasil. snaomi@uesb.br.

${ }^{3}$ Engenheira-Agrônoma, Mestre. Universidade Estadual do Sudoeste da Bahia, UESB. Estrada do Bem Querer, Km 04, Caixa Postal 95, 45000-000, Vitória da Conquista, Bahia, Brasil. lemoscarmem@yahoo.com.br.

${ }^{4}$ Engenheiro-Agrônomo, Doutor. Embrapa Agrobiologia, Laboratório de Micologia, BR 465, Km 7, C.P. (P.O. Box) 74505, 23890-000, Seropédica, Rio de Janeiro, Brasil. fdesouza@cnpab.embrapa.br 


\section{INTRODUÇÃO}

A associação de árvores com os cafeeiros resulta em vantagens como a redução dos valores extremos de temperatura (Pezzopane et al., 2005), proteção contra ventos, elevação da infiltração de água no solo (Narain et al., 1998), diminuição da erosão (Franco, 2000) e favorecimento da qualidade da bebida (Boulay et al., 2000).

Devido à bienalidade da produção e à oscilação do mercado cafeeiro, a possibilidade de agregação de renda adicional para o agricultor, por meio do consórcio com outra cultura, torna-se altamente favorável. Tal consorciação permite a geração de renda extra (Vieira et al., 2007) por meio de produtos como madeira, frutos, forragem e óleos essenciais. Os sistemas agroflorestais proporcionam tal condição, permitindo a exploração simultânea de um ou mais componentes arbóreos (Assis Júnior et al., 2003). Entretanto, é observada baixa produtividade de cafeeiros em sistemas sombreados decorrente de uma série de fatores como o excesso de sombreamento (Miranda et al., 1999), a utilização de espécies arbóreas inadequadas e o desconhecimento da otimização de manejo das árvores (Archanjo et al., 2007) e da nutrição mineral em sistemas agroflorestais (Jesus, 2008). São poucos os relatos de cafeeiros sob sombra com produção semelhantes à daqueles a pleno sol altamente tecnificados (Mello \& Guimarães, 2000). A maioria das experiências relatam produções extremamente baixas com relação à produção de cafezais a pleno sol em condições similares de cultivo (Campanha, 2001).

Apesar da grande diversidade de fatores afetados pela introdução do componente arbóreo no sistema de produção cafeeiro, o sombreamento é o tema em que as discussões principais mantêm foco. Embora muitos trabalhos tenham comprovado o efeito da restrição de radiação em características morfológicas (Morais et al., 2003; Righi, 2005; Lemos, 2008) edáficas (Corrêa da Silva, 2006; Salgado et al., 2006) e climáticas (Black \& Ong, 2000; Chirwa et al., 2007) de sistemas agroflorestais, pouco tem sido discutido sobre a radiação fotossinteticamente ativa incidente na planta e, muitas vezes, não há informações sobre a densidade do componente arbóreo. Frequentemente são verificados equívocos na interpretação da porcentagem de cobertura da área pelo estrato arbóreo e pela restrição da radiação incidente. Portanto, o esclarecimento específico das interações relacionadas à introdução do componente arbóreo deve ser realizado para que se possa integrá-lo à complexidade desses sistemas, estabelecendo formas de manejo cada vez mais refinadas. A compreensão dessa rede de interações poderá resultar em sustentabilidade ambiental e econômica para os cafeicultores.

Rev. Ceres, Viçosa, v. 57, n.1, p. 095-102, jan/fev, 2010
O presente trabalho teve como objetivo a comparação de características morfológicas dos cafeeiros e da conservação de umidade do solo em diferentes sistemas agroflorestais.

\section{MATERIAL E MÉTODOS}

A área do estudo localiza-se no município de Barra do Choça, no Sudoeste da Bahia, Planalto da Conquista, com latitude $14^{\circ} 51^{\prime}$ Sul e longitude $41^{\circ} 08^{\prime}$ Oeste, altitude média de 860 m e precipitação média anual de aproximadamente 800 mm (Figura 1).

A pesquisa foi realizada em propriedade particular sob regime de agricultura familiar. Há muitos anos a família conduz as lavouras de café no manejo orgânico. Essas estão situadas em áreas de relevo plano e cada sistema recebeu o manejo adequado às exigências de cultivo, que serão descritas mais detalhadamente a seguir. Não houve interferências quanto ao manejo adotado na propriedade.

O estudo foi realizado em três sistemas de cultivo de café (cafeeiro cultivado a pleno sol, associado ao vinhático, associado ao ingazeiro e ao abacateiro) e em uma mata nativa. As avaliações foram realizadas em duas épocas: março (época de elevada precipitação - estação úmida) e agosto (época com pouca precipitação - estação seca) de 2007. A amostragem foi constituída, em cada campo experimental, por quatro repetições para as variáveis de solo e seis para as demais. A unidade experimental foi definida por uma planta de café situada em disposição aleatória em local representativo do sistema. Os tratamentos foram comparados pelo teste "t" de Bonferroni, por meio do programa SISVAR (versão 5.0).

O sistema monocultura foi constituído por cafeeiros conduzidos em sistema tradicional (MONO). Essa lavoura foi implantada há sete anos, no espaçamento de 3 m x 1 $\mathrm{m}$, determinando uma densidade de 3.333 cafeeiros ha-1. Nesse sistema, o cafeeiro foi adubado em agosto de 2005, com $10 \mathrm{~L}$ de palha de café/cova, e roçado até quatro vezes por ano, sendo feita uma capina.

O Sistema Agroflorestal 1 (SAF-CV) foi formado pela associação do cafeeiro com o vinhático (Plathymenia spp.).

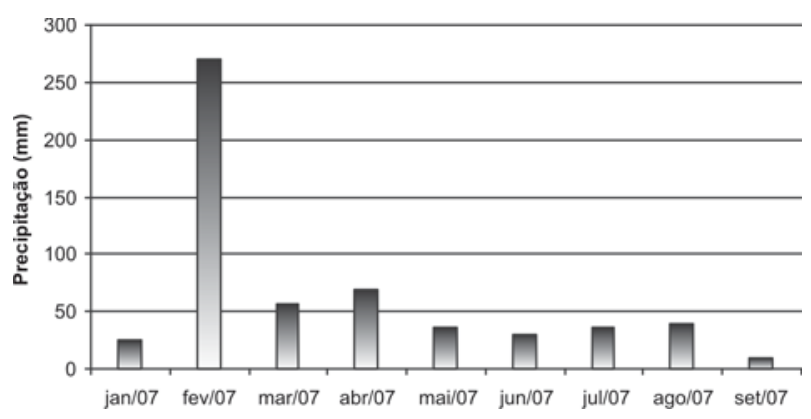

Figura 1. Precipitação pluvial mensal (mm) no período de janeiro a setembro de 2007, município Barra do Chaga, Bahia. 
Os cafeeiros foram plantados no espaçamento de 3 x 1,5 m, totalizando 2.222 cafeeiros ha-1. Essa lavoura fora implantada havia 23 anos, sendo realizada poda rasa nos cafeeiros (recepa); por ocasião das avaliações a rebrota estava com dois anos. A última adubação mineral nesse sistema foi realizada há 15 anos, sendo ele roçado uma vez por ano, sempre antes da colheita. A população de vinhático apresentava densidade de 1.255 árvores ha ${ }^{-1}$ e foi formada por meio de dispersão natural de uma árvore matriz que se localiza no interior da lavoura. O vinhático é uma espécie caducifólia, portanto sofre desfolha natural que, nas condições locais, se inicia em julho e estende-se até setembro, ocorrendo também uma abscisão de galhos.

No Sistema Agroflorestal 2 (SAF-CAI) o cafeeiro foi associado ao ingazeiro (Inga edulis) e ao abacateiro (Persea americana). A densidade do ingazeiro foi de 308 árvores.ha ${ }^{-1}$ e a do abacateiro, 175 árvores.ha ${ }^{-1}$, totalizando uma densidade arbórea de 483 árvores.ha-1 ${ }^{-1}$. Esse sistema foi implantado há 20 anos, e o cafeeiro recepado há sete anos em relação à coleta dos dados. Os cafeeiros foram mantidos no espaçamento de 3 x $1 \mathrm{~m}$, apresentando densidade de 3.333 plantas. ha-1 , e foram adubados há nove anos, sendo roçados uma vez por ano, antes da colheita. Não foram realizadas podas de manejo da sombra nas espécies arbóreas.

Foi utilizado um fragmento de mata nativa como referência para avaliação da umidade do solo. Esse fragmento fica próximo aos sistemas de cultivo de café. A vegetação predominante na região é do tipo Floresta Estacional Decidual (PMBC, 2008).

As avaliações foram realizadas em duas épocas diferentes e coincidentes com os seguintes estádios fenológicos do cafeeiro: maturação dos frutos (março de 2007) e pós-colheita (agosto de 2007).

Para a avaliação da radiação fotossinteticamente ativa e do nível de sombreamento dos sistemas utilizou-se um ceptômetro de barra (EMS-1, PPSystems, UK), constituído de um sensor com 0,80 m de comprimento, composto por 10 células fotossensíveis para determinar a Radiação Fotossinteticamente Ativa (RFA) Global, na rua e no interior da copa do cafeeiro. A RFA Global foi determinada em três posições: a pleno sol, a 1,5 m acima do nível do solo; na rua, também a 1,5 m acima do nível do solo entre duas linhas de café, e no interior da copa do cafeeiro, na altura do terço médio. Foram utilizadas seis repetições em cada posição, e os valores das radiações determinadas na rua e na copa do cafeeiro foram transformados em valores percentuais em relação à radiação global. O nível de sombreamento do sistema foi obtido subtraindo de 100 a porcentagem da radiação global avaliada na rua do cafeeiro: \% de sombreamento do sistema $=100-[($ PAR da rua/PAR global $) \times 100]$. A por- centagem de autossombreamento do cafeeiro foi calculada subtraindo de 100 a relação percentual da PAR que chega no interior da copa do cafeeiro em relação à PAR da rua: \% de autossombreamento $=100-$ [(PAR na copa/ PAR na rua) x 100]. Para determinar a umidade do solo, foram coletadas seis amostras em cada sistema, na profundidade de 0-20 cm, sob a projeção da copa do cafeeiro. Essas amostras foram misturadas, e homogeneizadas, formando-se em uma amostra composta de onde foram obtidas quatro repetições.

A umidade do solo foi avaliada pelo método gravimétrico. Foi determinado o peso de uma amostra do solo úmido em recipiente de peso conhecido e em balança analítica. Depois, a amostra foi deixada em estufa por $24 \mathrm{~h}$, a $105^{\circ} \mathrm{C}$. Após esse período, deixou-se esfriar em dessecador e pesou-se o recipiente com o solo seco. A umidade foi expressa em porcentagem correspondente à massa de solo. Para determinar os teores de umidade gravimétrica correspondentes ao ponto de murcha permanente (PMP) e à capacidade de campo (CC) utilizou-se equação formulada por Arruda et al. (1987). Em cada sistema foram coletadas amostras de solo rizosférico sob a projeção da copa de seis cafeeiros, na profundidade de $0-20 \mathrm{~cm}$. Esse solo foi homogeneizado, formando-se uma amostra composta, sendo retirados 500 g e encaminhados para análise física (Tabela 1). O solo coletado foi seco ao ar e peneirado para obtenção de terra fina seca ao ar, e, posteriormente, analisado no Laboratório de Solos da Universidade Estadual do Sudoeste da Bahia.

Para determinação da área foliar, foram coletadas 16 folhas, situadas no terceiro par de folhas, sentido basípeto do ramo, no terço médio da copa dos cafeeiros, sendo utilizado um integralizador de área ( LICOR , modelo LI3100). O diâmetro do caule foi avaliado no ramo ortotrópico (principal) por meio de um paquímetro, na altura do terço médio. O diâmetro da copa do cafeeiro foi avaliado na altura do terço médio e foi utilizada uma régua graduada colocada transversalmente ao ramo ortotrópico. A altura foi determinada com uma régua graduada disposta paralelamente ao caule, sendo a medida realizada entre o colo e o ápice do cafeeiro.

\section{RESULTADOS E DISCUSSÃO}

Em ambas as épocas de avaliação, os níveis de restrição de radiação dos SAFs foram intensos (Tabela 2). Tais valores mostraram-se desfavoráveis à produção de café em outros trabalhos. Conforme estudo realizado por SotoPinto et al. (2000), níveis de restrição entre 23 e 38\% da radiação incidente, em condições de arborização, foram favoráveis à produção dos cafeeiros, e partir de 50\% foi observada redução da produção. Lunz (2006), avaliando sistemas de café associado à seringueira, em diferentes 
Tabela 1. Análise física do solo rizosférico dos sistemas de produção de café solteiro (MONO), associado ao vinhático (SAF-CV) e ao abacateiro e ingazeiro (SAF-CAI) na profundidade de 0 a $20 \mathrm{~cm}$

\begin{tabular}{|c|c|c|c|c|c|}
\hline \multirow{3}{*}{$\begin{array}{l}\text { Sistema de } \\
\text { cultivo }\end{array}$} & \multirow{3}{*}{$\begin{array}{c}\text { Terra fina } \\
(<2,0 \mathrm{~mm})(\%)\end{array}$} & \multicolumn{4}{|c|}{ Composição granulométrica } \\
\hline & & $\begin{array}{l}\text { Areia grossa } \\
(0,2-2,0 \mathrm{~mm})\end{array}$ & $\begin{array}{c}\text { Areia fina } \\
(0,05-0,20 \mathrm{~mm})\end{array}$ & $\begin{array}{c}\text { Silte } \\
(0,002-0,05 \mathrm{~mm})\end{array}$ & $\begin{array}{c}\text { Argila } \\
(<0,002 \mathrm{~mm})\end{array}$ \\
\hline & & \multicolumn{4}{|c|}{ g/kg } \\
\hline \multicolumn{6}{|c|}{ Março/2007 } \\
\hline MONO & 100 & 340 & 200 & 100 & 360 \\
\hline SAF-CV & 100 & 390 & 170 & 60 & 380 \\
\hline SAF-CAI & 100 & 380 & 200 & 80 & 340 \\
\hline \multicolumn{6}{|c|}{ Agosto/2007 } \\
\hline MONO & 100 & 350 & 200 & 70 & 380 \\
\hline SAF-CV & 100 & 430 & 230 & 50 & 290 \\
\hline SAF-CAI & 100 & 400 & 220 & 70 & 310 \\
\hline
\end{tabular}

Tabela 2. Valores da radiação fotossinteticamente ativa (RFA) global na rua e no interior da copa do cafeeiro e níveis de sombreamento do sistema e de autossombreamento do cafeeiro, calculados com base nos valores das RFA global, na rua e no interior da copa do cafeeiro

\begin{tabular}{|c|c|c|c|c|c|c|c|c|c|c|}
\hline \multirow[t]{2}{*}{$\begin{array}{l}\text { Sistemas } \\
\text { de cultivo }\end{array}$} & \multicolumn{2}{|c|}{$\begin{array}{c}\text { RFAglobal } \\
\left(\mathbf{i m o l} . \mathbf{m}^{-2} \cdot \mathbf{s}^{-1}\right)\end{array}$} & \multicolumn{2}{|c|}{$\begin{array}{l}\text { RFA na rua do } \\
\text { cafeeiro } \\
\left(\text { ìmol. } \mathbf{m}^{-2} \cdot \mathrm{s}^{-1}\right)\end{array}$} & \multicolumn{2}{|c|}{$\begin{array}{l}\text { RFA no interior da } \\
\text { copa do cafeeiro } \\
\left(\text { ìmol.m-2.- } \mathrm{s}^{-1}\right)\end{array}$} & \multicolumn{2}{|c|}{$\begin{array}{c}\text { Sombreamento } \\
\text { do sistema } \\
(\%)\end{array}$} & \multicolumn{2}{|c|}{$\begin{array}{c}\text { Autossombreamento } \\
\text { do cafeeiro } \\
(\%)\end{array}$} \\
\hline & Março & Agosto & Março & Agosto & Março & Agosto & Março & Agosto & Março & Agosto \\
\hline MONO & 1787 & 345 & 1787 & 345 & 76 & 66 & $0,0 \mathrm{c}$ & 0,0 c & 95,8 aA & 73,9 abB \\
\hline SAF-CV & 1722 & 357 & 116 & 223 & 14 & 25 & 93,3 aA & 37,5 bB & 85,6 aA & 88,6 aA \\
\hline SAF-CAI & 395 & 507 & 69 & 53 & 17 & 17 & $82,3 \mathrm{bA}$ & 89,5 aA & $69,6 \mathrm{bA}$ & $61,4 \mathrm{bA}$ \\
\hline CV (\%) & - & - & - & - & - & - & 7,9 & 10,18 & 11,98 & 19,93 \\
\hline
\end{tabular}

Médias seguidas da mesma letra minúscula na coluna (tratamento) e maiúscula na linha (época) não diferem entre si pelo teste t de Bonferroni a $5 \%$.

níveis de sombreamento, verificou que níveis de sombreamento do cafeeiro entre 20 e $30 \%$ não afetaram sua produção. Nos dois SAFs, nas duas avaliações, os valores de Radiação Fotossinteticamente Ativa (RFA) na rua dos cafeeiros foram inferiores ao limite mínimo de saturação lumínica de 300 ìmol de fótons $\mathrm{m}^{-2} \cdot \mathrm{s}^{-1}$ para cafeeiros sombreados (Kumar \& Tieszen, 1980; Bote, 2007).

Nas duas épocas, as irradiâncias verificadas em MONO foram inferiores ao limite de fotoinibição da fotossíntese (2.200 ìmol. $\mathrm{m}^{-2} . \mathrm{s}^{-1}$, Nunes et al. (1993) citados por Morais et al. (2003) (Tabela 2). Entretanto, grande amplitude de variação foi observada entre as duas épocas, ocorrendo em março valores que atingiram $500 \%$ da RFA determinada em agosto. Em estudos realizados sobre cafeeiros associados às seringueiras, Oliveira et al. (2006) também verificaram variação sazonal da RFA, sendo os maiores valores observados no verão.

Na avaliação de março, foi observada em MONO RFA superior ao limite de saturação de luz (600 ìmol de fótons $\mathrm{m}^{-2} \cdot \mathrm{s}^{-1}$ para cafeeiros a pleno sol) definido por Bote (2007) e Kumar \& Tieszen (1980), e em agosto tais valores foram inferiores. Embora os limites de saturação lumínica e fotoinibição sejam frequentemente generalizados para os cafeeiros, Franck et al. (2007) postularam a existência de variações desses limites relacionados ao condicionamento das plantas aos gradientes de RFA que ocorrem no ambiente de cultivo. No referido estudo, foi verificada relação linear crescente entre níveis de sombreamento e limites de saturação lumínica. Portanto, apesar de muitos estudos sobre arborização de cafezais expressarem a intensidade de radiação em termos relativos de porcentagem de restrição luminosa, a informação sobre valores da densidade de fluxo de fótons incidente é de extrema importância para a avaliação do comportamento fotossintético dos cafeeiros. Jaramillo-Botero (2007), em estudos sobre arborização de cafeeiros, observou valor anual médio de radiação incidente no local do experimento de 228 ìmol de fótons. $\mathrm{m}^{-2} . \mathrm{s}^{-1}$ nas condições de Viçosa, MG. Oliveira et al. (2006) verificaram valores de 1.100 a 2.200 ìmol de fótons. $\mathrm{m}^{-2} \cdot \mathrm{s}^{-1}$, determinados no inverno e verão, respectivamente, durante o período das 11 às $13 \mathrm{~h}$, em Lavras, Minas Gerais. 
É necessário pontuar que além da radiação luminosa, o sombreamento pode afetar outros fatores ambientais como a redução do déficit de pressão de vapor de água e da variação de temperatura do ar (Oliveira et al., 2006). Tais alterações podem afetar características do cafeeiro como a arquitetura da planta (Morais et al., 2003; Righi, 2005; Lemos, 2008), condutância estomática (Nascimento et al., 2006; Gomes et al., 2008), temperatura da folha (Morais et al., 2007; Nascimento et al., 2006) e carga de frutos (Ricci et al., 2006; Pezzopane et al., 2007), resultando em forte interação com a radiação incidente e determinando a capacidade fotossintética da planta (Nascimento et al., 2006; Gomes et al., 2008).

Em março, foi observado maior nível de restrição de luz para o sistema café e vinhático, seguido pelo sistema café associado ao abacate e ao ingá em relação ao sistema de cultivo de café a pleno sol (Tabela 2). Na avaliação realizada em agosto, o maior nível de sombreamento foi verificado para SAF-CAI, seguido do SAF-CV, em relação ao MONO (Tabela 2). Na avaliação houve decréscimo acentuado no nível de sombreamento de SAFCV. , devido à abscisão de folhas. Como o componente arbóreo em SAF-CV foi constituído apenas pelo vinhático, uma espécie caducifólia (Goulart et al., 2005), essa sofreu desfolha total e abscisão de galhos, elevando a incidência de luz no sistema, reduzindo o sombreamento de 93,3 para 37\%.

Na avaliação de março, os maiores índices de autossombreamento foram verificados para MONO $(95,8 \%)$ e SAF-CV (85,6\%), sendo ambos considerados semelhantes (Tabela 2). Fernandes (1986) relacionou baixos valores de autossombreamento à ocorrência de internódios mais longos e menor número de folhas, os quais caracterizam uma adaptação da planta ao ambiente sombreado com a finalidade de aumentar a eficiência fotossintética (Rena et al., 1994). Tal adaptação pode ter sido a explicação para que cafeeiros cultivados a pleno sol e em SAF$\mathrm{CV}$ tenham apresentado copa mais densa do que no SAFCAI no presente trabalho. As diferentes fases de desenvolvimento dos cafeeiros nos dois sistemas resultaram na ocorrência de alterações morfológicas específicas, condicionando maior nível de auto-sombreamento em SAF-CV. Convém lembrar que os cafeeiros de SAF-CV sofreram poda drástica dois anos antes do referido estudo e, por isso, apresentaram características morfológicas típicas de desenvolvimento inicial da cultura.

Para a avaliação de autossombreamento realizada em agosto, o comportamento entre os SAFs foi semelhante ao verificado em março, sendo mantido o maior valor em SAF-CV (88,6\%) em relação a SAF-CAI (61,4\%). Entretanto, para cafeeiros mantidos a pleno sol, foi observada redução do índice de autossombreamento, mantendo valor intermediário entre os SAFs. A avaliação realizada em agos- to coincidiu com o período pós-colheita do café, sendo o efeito da desfolha de origem física. Esse efeito foi acentuado nos cafeeiros mantidos a pleno sol, devido aos processos de colheita realizados nos meses anteriores. Em estudo realizado por Ricci et al. (2006), maior susceptibilidade à abscisão foliar promovida pela colheita em relação à condição de sombreamento natural foi observada em cafeeiros a pleno sol.

O cafeeiro apresentou maior altura em sistema SAFCAI em relação aos MONO e SAF-CV nas duas avaliações (Tabela 3). A maior altura do cafeeiro cultivado em SAF-CAI foi resultado do elevado nível de sombreamento nesse sistema, corroborando com outros estudos realizados com cafeeiros sombreados (Fahl et al., 1994).

No presente estudo, desenvolvido em condição de campo com plantas adultas, foi observada maior altura dos cafeeiros em condição de sombreamento. A diferença entre os estádios de desenvolvimento, aliada às condições diferenciadas de sombreamento, foi fator determinante para tal divergência de comportamento entre situações, em princípio definido como semelhante. Embora em muitos estudos a característica altura seja utilizada como um dos parâmetros de crescimento, Da Matta (2004) ressalta que em sistemas com restrição de luz, esta é uma alteração morfológica que não pode ser diretamente relacionada ao vigor vegetativo da planta e sim a uma anomalia fisiológica denominada estiolamento.

Os maiores valores de diâmetro do caule foram observados em MONO e SAF-CAI, quando comparados ao SAF-CV, nas avaliações das duas épocas (Tabela 3). A semelhança de valores de diâmetro do caule em MONO e SAF-CAI pode indicar similaridade na síntese de carboidratos estruturais neste órgão. O menor diâmetro do caule em SAF-CV, nas duas avaliações, ocorreu em função do ciclo do cafeeiro ter sido alterado pela recepa. Desse modo, o cafeeiro de SAF-CV, em comparação a MONO e SAF-CAI, não havia atingido seu máximo desenvolvimento vegetativo.

Devido ao fato de a lavoura ter sofrido recepa total e ter sido avaliada após dois anos de rebrota, menores valores da altura e do diâmetro do caule do cafeeiro foram observados em SAF-CV. De acordo com Ricci et al. (2006), a taxa de crescimento dos cafeeiros conduzidos em campo sob sombreamento é reduzida no início do desenvolvimento da cultura (primeiros 15 meses).

Na avaliação realizada em março, não houve diferença no diâmetro da copa do cafeeiro entre os sistemas, sugerindo que MONO (pleno sol) e SAFs (sombreados) produziram ramos de comprimento semelhante. Em agosto, maior diâmetro de caule foi verificado em SAF-CAI, valor intermediário foi observado em SAF-CV e MONO apresentou menor diâmetro. 
Tabela 3. Caracterização morfológica do cafeeiro (altura, diâmetro do caule, diâmetro da copa e área foliar) em sistema de cultivo de café a pleno sol (MONO) e dois sistemas agroflorestais (SAF-CV e SAF-CAI)

\begin{tabular}{|c|c|c|c|c|c|c|c|c|}
\hline \multirow{3}{*}{$\begin{array}{l}\text { Sistemas } \\
\text { de cultivo }\end{array}$} & \multicolumn{8}{|c|}{ Morfologia do cafeeiro } \\
\hline & \multicolumn{2}{|c|}{$\begin{array}{c}\text { Altura } \\
(\mathbf{m})\end{array}$} & \multicolumn{2}{|c|}{$\begin{array}{l}\text { Diâmetro do caule } \\
\text { (cm) }\end{array}$} & \multicolumn{2}{|c|}{$\begin{array}{c}\text { Diâmetro da copa } \\
\text { (m) }\end{array}$} & \multicolumn{2}{|c|}{$\begin{array}{l}\text { Área foliar individual } \\
\qquad\left(\mathrm{cm}^{2}\right)\end{array}$} \\
\hline & Março & Agosto & Março & Agosto & Março & Agosto & Março & Agosto \\
\hline MONO & $2,35 \mathrm{~b}$ & $2,51 \mathrm{~b}$ & 2,37 a & $2,86 \mathrm{a}$ & $1,65 \mathrm{a}$ & $1,71 \mathrm{~b}$ & $38,65 \mathrm{~b}$ & $41,71 \mathrm{~b}$ \\
\hline SAF-CV & $2,07 \mathrm{~b}$ & $2,22 b$ & $1,78 \mathrm{~b}$ & $1,68 \mathrm{~b}$ & $1,80 \mathrm{a}$ & $1,76 \mathrm{a}$ & 75,63 a & 77,12 a \\
\hline SAF-CAI & $2,90 \mathrm{a}$ & 3,03 a & $2,37 \mathrm{a}$ & $2,61 \mathrm{a}$ & $1,86 \mathrm{a}$ & $2,05 \mathrm{a}$ & 69,81 a & $63,21 \mathrm{a}$ \\
\hline $\mathrm{CV}(\%)$ & 13,17 & 8,05 & 12,32 & 17,99 & 13,37 & 11,72 & 12,66 & 20,63 \\
\hline
\end{tabular}

Médias seguidas da mesma letra minúscula na coluna (tratamento) não diferem entre si pelo teste t de Bonferroni a 5\%.

Maior área foliar individual (AFI) foi verificada nos SAFs em relação ao MONO nas duas avaliações (Tabela 3). Em SAF-CV, a expansão da AFI foi de 90\% (março) e 85\% (agosto) superior à do café em MONO. Em SAF-CAI, foi verificado AFI de $80 \%$ (março) e 51\% (agosto) superior em relação ao MONO. Houve aumento de $15 \%$ na AFI do cafeeiro em SAF-CV em relação ao SAF-CAI. Maiores índices de autossombreamento verificados em SAF-CV potencializaram o efeito do sombreamento neste sistema quando comparado ao SAF-CAI. Em estudo realizado por Morais et al. (2003), aumento no tamanho individual das folhas dos cafeeiros foi uma das principais alterações morfológicas observadas em sistemas arborizados. Foi verificado menor peso de massa seca e menor massa específica (massa/área), resultando em maior eficiência da interceptação da energia solar disponível no sistema. Portanto, a folha foi o órgão que apresentou grande capacidade de adaptação à restrição de luz dos SAFs, o que pode resultar em maior eficiência de captação da energia solar disponível (Fahl et al., 1994).

Na avaliação realizada em março, época chuvosa, não houve diferença entre a umidade gravimétrica do solo (US) para os três sistemas de cultivo de café e mata nativa (Tabela 4).
Em agosto, época caracterizada por menores índices de precipitação, US foi superior em MONO (17,3\%), seguido por SAF-CV (15,1\%) e SAF-CAI (9,5\%) (Tabela 4). Os menores valores de US dos SAFs provavelmente foram decorrentes da soma da transpiração dos cafeeiros com o componente arbóreo. Resultados similares são relatados por Neves et al. (2007) em trabalhos realizados com cafés arborizados na estação seca, em Viçosa, MG. Esses autores verificaram que a introdução do componente arbóreo elevou o potencial de transpiração dos sistemas agroflorestais, resultando na rápida e intensa redução de US em relação ao sistema a pleno sol. Matsumoto et al. (2006), em estudo realizado durante três anos em sistema de cultivo de café e grevilea, em Vitória da Conquista-BA, com avaliações trimensais de US do solo, verificaram que as variações de US foram dependentes da distribuição espacial horizontal das amostras em relação ao componente arbóreo e da profundidade do solo. Neste estudo, a US dos sistemas foi mantida, nas duas épocas, acima do Ponto de Murcha Permanente (PMP) (Tabela 4). Entretanto, devido ao elevado potencial de transpiração ocorrido em agosto para SAF-CAI em relação aos demais, US foi mantida próxima ao PMP.

Tabela 4. Umidade gravimétrica do solo (US) rizosférico de cafeeiros em monocultivo (MONO), sistema agroflorestal com vinhático (SAF-CV) e sistema agroflorestal com abacateiro e ingazeiro (SAF-CAI) na profundidade de $0-20 \mathrm{~cm}$

\begin{tabular}{|c|c|c|c|c|c|c|}
\hline \multirow{3}{*}{$\begin{array}{l}\text { Sistema de } \\
\text { cultivo }\end{array}$} & \multicolumn{6}{|c|}{ Umidade gravimétrica(\%) } \\
\hline & \multicolumn{3}{|c|}{ Março } & \multicolumn{3}{|c|}{ Agosto } \\
\hline & $\mathbf{P M P}^{1}$ & $\mathrm{CC}^{2}$ & US & PMP & $\mathbf{C C}$ & US \\
\hline MONO & 10,7 & 21,3 & 19,0 a & 11,2 & 22,1 & $17,3 \mathrm{a}$ \\
\hline SAF-CV & 11,2 & 22,1 & 14,5 a & 8,6 & 18,5 & $15,1 \mathrm{~b}$ \\
\hline SAF-CAI & 10,1 & 20,6 & 18,2 a & 9,2 & 19,3 & $9,5 \mathrm{~d}$ \\
\hline MATA & 9,5 & 19,7 & 18,5 a & 11,5 & 22,5 & $12,7 \mathrm{C}$ \\
\hline CV (\%) & - & - & 18,9 & - & - & 2,04 \\
\hline
\end{tabular}

Médias seguidas de mesma letra na coluna (tratamento) não diferem entre si a 5\% de probabilidade pelo teste t de Bonferroni.

1 - Ponto de murcha permanente (PMP).

2 - Capacidade de campo (CC).

Rev. Ceres, Viçosa, v. 57, n.1, p. 095-102, jan/fev, 2010 


\section{CONCLUSÕES}

Os sistemas de cultivo definem os parâmetros morfológicos avaliados do cafeeiro.

Os sistemas agroflorestais causam grande sombreamento para o cafeeiro, resultando em plantas com elevada área foliar individual e diâmetro da copa nas épocas de pouca precipitação e úmida.

O vinhático resulta em grande sazonalidade no nível de sombreamento dos cafeeiros, que apresentam maior valor em março (93,3\%), época úmida, e menor em agosto (37,5\%), época de pouca precipitação.

O monocultivo de café apresenta maior capacidade de conservação de água no solo na época de menor precipitação.

\section{AGRADECIMENTOS}

À Fundação de Amparo à Pesquisa do Estado da Bahia (FAPESB), pela concessão da bolsa de estudo ao primeiro autor.

\section{REFERÊNCIAS}

Archanjo KMPA, Jesus Junior WC \& Pezzopane JEM (2007) Respostas ecofisiológicas de cafeeiros em sistemas agroflorestais. Revista Brasileira de Agroecologia, 2:702-705.

Arruda FB, Zullo J \& Oliveira JB (1987) Parâmetros de solo para cálculo da disponibilidade de água com base na textura. Revista Brasileira de Ciência do Solo, 11:11-15.

Assis Júnior SL, Zanuncio JC, Kasuya MCM, Couto L \& Melido RCN (2003) Atividade microbiana do solo em sistemas agroflorestais, monoculturas, mata natural e área desmatada. Revista Árvore, 27:35-41.

Black CR \& Ong CK (2000) Utilization of light and water in tropical agriculture. Agriculture Forest Meteorology, 104:25-47

Bote AD (2007) Physiological effect of shade on growth and production of organic coffee in Ethiopia. Thesis of master science. Wageningen University, Wageningen, 67p

Boulay M, Somarriba E \& Olivier A (2000) Calidad de Coffea arabica bajo sombra de Erythrina poeppigiana a diferentes elevaciones en Costa Rica. Agroforestería en las Américas, $7: 40-42$.

Campanha MM (2001) Análise comparativa de cafeeiros (Coffea arabica L) em sistema agroflorestal e monocultivo na Zona da Mata de Minas Gerais. Tese de Doutorado. Universidade Federal de Viçosa, Viçosa, 132p.

Chirwa PW, Ong CK, Maghembe J \& Black CR (2007) Soil water dynamics in cropping systems containing Gliricidia sepium, pipeonpea and maize in southern Malawi. Agroforest Systems, 69:29-43.

Corrêa da Silva MS (2006) Indicadores de qualidade do solo em sistemas agroflorestais em Paraty-RJ. Dissertação de Mestrado. Universidade Federal Rural do Rio de Janeiro, Seropédica, 54p.

Da Matta FM (2004) Fisiologia do cafeeiro em sistemas arborizados. In: Matsumoto SN (Org.) Arborização de cafezais no Brasil. Vitória da Conquista, Ed. UESB. p.87-122.
Fahl JI, Carelli MLC, Vega J \& Magalhãe AC (1994) Nitrogen and irradiance levels affecting net photosynthesis and growth of young coffee plants (Coffea arabica L.). Journal of Horticultural Science, 69:161-169.

Fernandes DR (1986) Manejo do cafezal. In: Rena AB, Malavolta E, Rocha M \& Yamada T (Eds.) Cultura do café: fatores que afetam a produtividade. Piracicaba, Potafós. p.275-300.

Franck N, Vaast P \& Dauzat J (2007) What limits photosynthesis of coffee leaves under sun and shade? In: Second International Symposium on Multi-Strata Agroforestry System with Perennial Crops, Turrialba, Anais. CATIE. CD-ROM.

Franco FS (2000) Sistemas agroflorestais: uma contribuição para a conservação dos recursos naturais na zona da mata de Minas Gerais. Tese de Doutorado. Universidade Federal de Viçosa, Viçosa, 148p.

Gomes IAC, Castro EM, Soares AM, Alves JD, Alvarenga MIN, Alves E, Barbosa JPRAD \& Fries DD (2008) Alterações morfofisiológicas em folhas de Coffea arabica L. cv. "Oeiras" sob influência do sombreamento por Acacia mangium Willd. Ciência Rural, 38:109-115.

Goulart MF, Lemos-Filho JP \& Lovato MB (2005) Phenological variation within and among populations of Plathymenia reticulate in Brazilian Cerrado, the Atlantic Forest ant Transitional Sites. Annals of Botany, 96:445-455.

Jaramillo-Botero C (2007) Resposta de cafeeiros ao sombreamento e à dinâmica de serrapilheira em condições de sistema agroflorestal. Tese de doutorado. Universidade Federal de Viçosa, Viçosa, 72p.

Jesus J (2008) Atributos do solo e da nutrição do cafeeiro em sistema agroflorestal e monocultivo. Tese de Doutorado. Escola Superior de Agricultura “Luiz de Queiroz”, Piracicaba, 147p.

Kumar D \& Tieszen LL (1980) Photosynthesis in Coffea arabica. I. Effects of light and temperature. Experimental Agriculture, 16:13-19.

Lemos CL (2008) Características morfo-fisiológicas e assimilação de nitrogênio em cafeeiros em sistema a pleno sol e associados com abacateiro (Persea americana) e ingazeiro (Inga edulis) em Barra do Choça, Bahia. Dissertação de Mestrado. Universidade Estadual do Sudoeste da Bahia, Vitória da Conquista, 94p.

Lunz AMP (2006) Crescimento e produtividade do cafeeiro sombreado e a pleno sol. Tese de doutorado. Escola Superior de Agricultura "Luiz de Queiroz", Piracicaba, 94p.

Matsumoto SN, Faria GO, Viana AES, Rocha VS \& Novaes AB (2006) Water relations in a coffee grove planted with grevilleas in Vitória da Conquista-Bahia, Brazil. Coffee Science, 1:71-83.

Mello JT \& Guimarães DP (2000) A cultura do café em sistemas consorciados na região do Cerrado. In: I Simpósio de Pesquisa dos Cafés do Brasil. Poços de Caldas. Resumos expandidos. Embrapa. p.963-966.

Miranda EM, Pereira RCA \& Bergo CL (1999) Comportamento de seis linhagens de café (Coffea arabica L.) em condições de sombreamento e a pleno sol no estado do Acre, Brasil. Ciência e Agrotecnologia, 23:62-69.

Morais H, Caramori PH, Ribeiro AMA, Gomes JC \& Koguishi MS (2007) Microclimatic characterization and productivity of coffee plants grown under shade of pigeon pea in Southern Brazil. Pesquisa Agropecuária Brasileira, 41:763-770.

Morais H, Marur CJ, Caramori PH, Ribeiro AMA \& Gomes JC (2003) Características fisiológicas e de crescimento de cafeeiro sombreado com guandu e cultivado a pleno sol. Pesquisa Agropecuária Brasileira, 38:1131-1137. 
Narain P, Singh RK, Sindhwal NS \& Joshie P (1998) Water balance and water efficiency of different land uses in western Himalayan valley region. Agriculture Forest Meteorology, 37:225-240.

Nascimento EA, Oliveira LEM, Castro EM, Delú Filho N, Mesquita AC \& Vieira CV (2006) Alterações morfofisiológicas em folhas de cafeeiro (Coffea arabica L.) consorciado com seringueira (Hevea brasiliensis Muell. Arg.). Ciência Rural, 36:852857.

Neves YP, Martinez HEP, Souza CM \& Cecon PR (2007) Teor de água e fertilidade do solo com cafeeiros cultivados em sistemas agroflorestais. Revista Árvore, 31:575-588.

Oliveira CRM, Barbosa JPRAD, Soares AM, Oliveira LEM \& Macedo RLG (2006) Trocas gasosas de cafeeiros (Coffea arabica L.) e seringueiras (Hevea brasiliensis Muell. Arg.) em diferentes sistemas de cultivo na região de larvas, MG. Revista Árvore 30:197-206

Pezzopane JRM, Pedro Junior MJ \& Gallo PB (2005) Radiação solar e saldo de radiação em cultivo de café a pleno sol e consorciado com banana 'prata anã'. Bragantia, 64:485-497.

Pezzopane JRM, Pedro Júnior MJ, Gallo PB, Camargo MBP \& Fazuoli LC (2007) Avaliações fenológicas e agronômicas em café arábica cultivado a pleno sol e consorciado com banana 'prata anã'. Bragantia, 66:701-709.
PMBC Prefeitura Municipal de Barra do Choça (2008) Vegetação. Disponível em: <http://www.barradochoca.ba.gov.br/>. Acessado em: 6 de outubro de 2008 .

Rena AB, Nacif AP \& Pereira AA (1994) Fisiologia do cafeeiro em plantios adensados. In: Simpósio Internacional Sobre Café Adensado, Londrina. Anais. Iapar. p.73-85.

Ricci M dos SF, Costa JR, Pinto AN \& Santos VLSS (2006) Cultivo orgânico de cultivares de café a pleno sol e sombreado. Pesquisa Agropecuária Brasileira, 41:569-575.

Righi CA (2005) Avaliação ecofisiológica do cafeeiro (Coffea arabica L) em sistema agroflorestal e em monocultivo. Tese de Doutorado. Escola Superior de Agricultura “Luiz de Queiroz”, Piracicaba, 101p.

Salgado BG, Macedo RLG, Alvarenga MIN \& Venturin N (2006) Avaliação da fertilidade dos solos de sistemas agroflorestais com cafeeiro (Coffea arabica L) em Lavras-MG. Revista Árvore, 30:343-349.

Soto-Pinto L, Perfecto I \& Caballero-Nieto J (2000) Shade over coffee: its effects on berry borer, leaf rust and spontaneous herbs in Chiapas, México. Agroforestry Systems, 55:37-45.

Vieira TA, Rosa LS, Vasconcelos PCS, Santos MM \& Modesto RS (2007) Adoção de sistemas agroflorestais na agricultura familiar, em Igarapé-Açu, Pará, Brasil. Revista de Ciências Agrárias, 9-22. 